\title{
Investigation on tool function and removal characteristics for bonnet surfacing
}

\author{
Yunpeng Feng ${ }^{1,}$, , Haobo Cheng ${ }^{1,2}$ \\ ${ }^{1}$ School of Optoelectronics, Beijing Institute of Technology, 5 South Zhongguancun Street, Beijing, China \\ ${ }^{2}$ Shenzhen research Institute, Beijing Institute of Technology, Shenzhen, China
}

\begin{abstract}
Since computer controlled optical surfacing (CCOS) processes have been proposed in the 1960s, many processes are developed for precision optics successfully. In this present work, a novel approach, the precessions process, is proposed and used for large segments fabrication. The removal function of bonnet polisher based on velocity and pressure distribution, which are obtained from the geometry of the process tool-motion and Hertzian contact theory respectively, are simulated. A dynamic finite element analysis (FEA) model is constructed to optimize process parameters. At last, detailed experimental studies are carried out to verify the optimal parameters.
\end{abstract}

\section{Introduction}

With rapid development of science and technology, the demands of precision optical elements with strict specifications, including surface figure, subsurface quality, power spectrum density and production efficiency, have increased fast. Conventional polishing is done mainly by skilful workers using specialized tools, which is not feasible for precision products.

Therefore, computer controlled optical surfacing (CCOS) processes have been developed for fabrication of precision optics since the 1960s [1-5]. These processes have radically different polishing mechanisms, including computer controlled polishing [6], ion beam figuring (IBF) [7], plasma-assisted chemical etching [8], magnetorheological finishing [9], magnetorheological jet polishing [10,11]. Many large aspheric even off-axis segments have been successfully fabricated using them [4, 5].

A novel approach, the precessions polishing process, proposed by D.D. Walker group [4], was a type of subaperture technology. A tool is comprised of an inflated, bulged rubber membrane of spherical form (bonnet), and covered with standard flexible polishing cloths. The standard polishing slurries was used in this process. Precession motion was induced to produce a mathematically well-behaved near-Gaussian influence function [12]. The bonnet polisher rocked about its pole through precise $\mathrm{CNC}$ control of the position and orientation of a spinning. Recently, the process was successfully applied to control the edge zoom of hexagonal segments fabrication $[13,14]$.

In this paper, we discussed the removal function of bonnet polisher. The velocity and pressure distribution were obtained through the geometry of the process toolmotion and Hertzian contact theory respectively. A dynamic finite element analysis (FEA) model is used to analyse the used parameters. At last, detailed experimental studies verify the optimal parameters.

\section{Mathematical model of polishing process with bonnet polisher}

It is crucial for embedded process control intelligence (i.e. optimization software) to characterize the polishing process mathematically, and Preston hypothesis [15] is a basic classical model for optical polishing process, as shown in Eq. (1). It is suitable for many sub-aperture technologies, which remove materials purely by the mechanical effects.

$$
\Delta z=\kappa P(x, y) V(x, y) \Delta t(x, y)
$$

where $\Delta z$ denotes the material removal depth; $\kappa$ is the process-dependent coefficient related to the work piece material, polishing-tool, polishing slurry and temperature of work environment et al.; $P(x, y)$ and $V(x, y)$ are the pressure and the relative velocity distribution between tool and optic, respectively; $\Delta t$ is the working time of the tool staying on the optic.

The removal depth $\Delta d$ at a given point can be expressed as the convolution of unit removal function $r(x, y)$ and dwell time map $t(x, y)$, as shown in Eq.(2). $\otimes$ is the dimensional convolution operator.

$$
\Delta d(x, y)=r(x, y) \otimes t(x, y)
$$

According to surface errors measured by interferometer or other instruments, modern deterministic polishing may create dwell map by deconvolution, thereby the error will be eliminated completely.

\footnotetext{
${ }^{a}$ Corresponding author: feng_yp@126.com
} 


\section{The tool function of bonnet polisher}

The tool function of bonnet polisher can be determined, provided the velocity and the pressure distribution can be obtained based on given working condition. The bonnet with spherical form rotated around its axis, and the rotation axis rocked around the normal of the surface being polished. Figure 1 shows the diagram of the movement of bonnet polisher. Where $S_{\text {area }}$ is the contacted circle area, $O$ is the centre of $S_{\text {area }}, R$ is the radius of bonnet; $M N$ is the rotation axis of bonnet, and the rotation speed is $\omega_{0}$; The bonnet also rotate about the $Z$-axis with $\omega_{1} ; \theta$ is the Precession Angle between two axes.

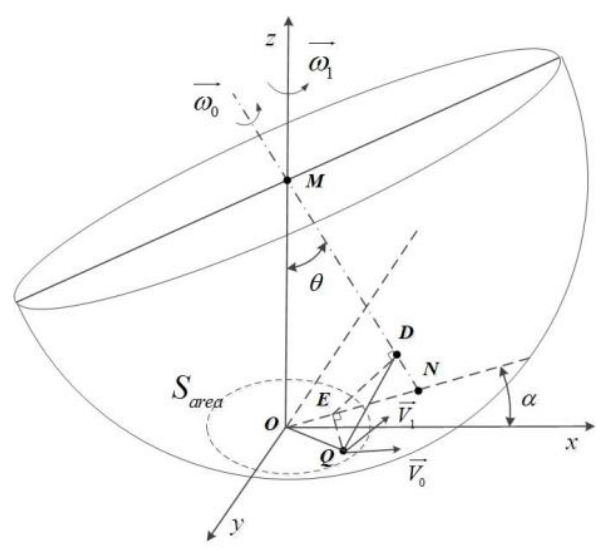

Figure 1. Diagram of movement of bonnet polisher, the dashed line

\subsection{Calculation of relative velocity distribution}

In the work presented here, the relative velocity distribution is obtained according to the geometry of the process tool-motion. The velocity relation of any point $(Q)$ in the polishing contact zone includes linear velocity $\vec{V}_{0}$ and $\vec{V}_{1} \cdot \vec{V}_{0}$ is the linear velocity rotating about $M N$ axis, and $\vec{V}_{1}$ is the linear velocity rotating about $Z$-axis. $h$ is the compression values, and $R$ is the radius of bonnet.

From known conditions, the coordination of point $M$ is $(0,0, R-h)$, and the coordination of point $N$ is

$$
\begin{gathered}
\left\{\begin{array}{c}
N_{x}=(R-h) \tan \theta \cos \alpha \\
N_{y}=(R-h) \tan \theta \sin \alpha . \\
N_{z}=0
\end{array}\right. \\
\text { Then } O E=r \cdot \cos (\alpha+\pi-\varphi), O N=(R-h) \tan \theta .
\end{gathered}
$$

Suppose the polar angle of point $Q$ is $\varphi(0 \leq \varphi \leq 2 \pi)$, and $\tan \varphi=y / x, \quad r=\sqrt{x^{2}+y^{2}}$, the coordination of point $E$ is

$$
\left\{\begin{array}{c}
E_{x}=r \cos (\varphi-\alpha) \cos \alpha \\
E_{y}=r \cos (\varphi-\alpha) \sin \alpha \\
E_{z}=0
\end{array}\right.
$$

The coordination of point $D$ is

$$
\left\{\begin{array}{c}
D_{x}=\left((O N-O E) \cos ^{2} \theta+O E\right) \cos \alpha \\
D_{y}=\left((O N-O E) \cos ^{2} \theta+O E\right) \sin \alpha \\
D_{z}=(O N-O E) \cos \theta \sin \theta
\end{array} .\right.
$$

At last, the linear velocity $\vec{V}_{0}$ and $\vec{V}_{1}$ can be obtained, respectively, as shown in Eq.(6) .

$$
\overrightarrow{V_{0}}=\frac{\overrightarrow{N M} \times \overrightarrow{D Q}}{|\overrightarrow{N M}|} \vec{\omega}_{0}, \overrightarrow{V_{1}}=\frac{\overrightarrow{O M} \times \overrightarrow{O Q}}{|\overrightarrow{O M}|} \vec{\omega}_{1}
$$

where $\overrightarrow{N M}=M-N, \overrightarrow{D Q}=Q-D, \overrightarrow{O Q}=Q-O$. The velocity vector of point $Q$, a space vector changing with its coordination (point $Q$ ), is $\vec{V}=\overrightarrow{V_{0}}+\overrightarrow{V_{1}}$. Because the motion of point $Q$ was constrained in contacted area, normally its projection in the $x-y$ plane was used during calculation. The relative velocity of $Q$ can be expressed by Eq.(7),

$$
V_{Q}=\sqrt{V_{x}^{2}+V_{y}^{2}}
$$

where $V_{x}$ and $V_{y}$ are the components of the vector $(\vec{V})$.

\subsection{The pressure distribution}

The radius of contact polishing area is much less than that of bonnet, and the contact problem between bonnet and surface was simplified to that between the sphere and flat. The pressure in contact area $S_{\text {area }}$ include: the internal pressure $p_{0}$ of bonnet, the pressure $p_{1}$ due to elastic deformation of the bonnet, and the pressure $p_{2}$ due to centrifugal distortion while the bonnet rotate about its axis. During polishing, $p_{0}$ is an invariant constant.

According to Hertzian contact theory, the pressure distribution fits the parabolic distribution as shown in Eq.(8). Figure 2 depicts the diagram of bonnet deformation

$$
p_{1}=\frac{2 E^{*} h}{\pi a}\left(1-\frac{x^{2}}{2 h R}\right)^{\frac{1}{2}}
$$

where $R$ is the radius of bonnet, $h$ is the compression value, $a=\sqrt{2 R h}$ is the radius of the contact area $\frac{1}{E^{*}}=\frac{1-v_{1}^{2}}{E_{1}}+\frac{1-v_{2}^{2}}{E_{2}}, E_{1}$ and $E_{2}$ is Young's modulus of the bonnet and glass respectively, $v_{1}$ and $v_{2}$ is the Poisson ratio of the bonnet and glass, respectively. 
Suppose the mass of a volume element locating at point $Q$ in membrane is $m$, the centrifugal force is

$$
F=m \cdot \overline{F Q} \cdot \omega_{0}^{2},
$$

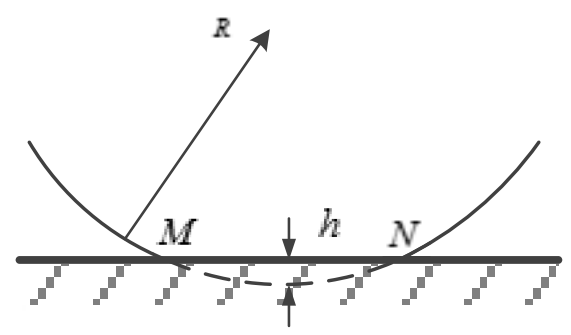

(a)

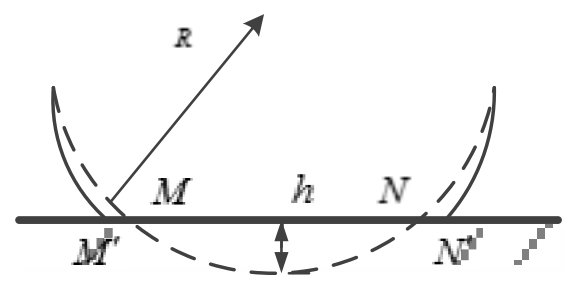

(b)

Figure 2. Diagram of bonnet (a) before and (b) after deformation, dash line denotes un-deformed membrane and solid line denotes deformed membrane

where $\omega_{0}$ is the rotation speed, $m=\rho \cdot \Delta s \cdot \Delta h, \Delta s \cdot \Delta h$ is the volume of the element, $\rho$ is the density of membrane. The pressure $p_{2}$ can be expressed as

$$
\begin{aligned}
p_{2} & =\frac{F}{S}=\frac{\overline{F Q} \cdot \omega_{0}^{2} \cdot \rho \cdot \Delta s \cdot \Delta h \cdot \sin \gamma}{\Delta s} \\
& =\overline{F Q} \rho \omega_{0}^{2} \sin \gamma
\end{aligned}
$$

Where $\gamma$ denotes the angle between $F Q$ and the $X-Y$ plane.

\subsection{The removal function}

The tool removal function depends on the relative velocity and pressure distribution mainly, and other conditions which was considered as the processdependent coefficient. There are three working modes based on processing attitude, plumb processing mode, tilt processing mode, and precession processing mode. Actually for plumb processing mode, the material removal of the centre of polishing spot is zero, and the profile of the tool function looks like $\mathrm{V}$ shape. It is not commonly used for fabrication.

\section{(1) Tilt processing mode}

There is a constant tilt angle between the rotating axis of bonnet and the normal line of the polishing area. The contact area is ellipse. Based on the above analysis, the tool function of tilt mode is shown in Figure 3. The following parameters were applied in simulation: the radius of bonnet is $40 \mathrm{~mm}$, the compression is $0.3 \mathrm{~mm}$, the thick of membrane is $1 \mathrm{~mm}$, the rotation speed is $120 \mathrm{rpm}$, and the tilt angle is 20 degree.

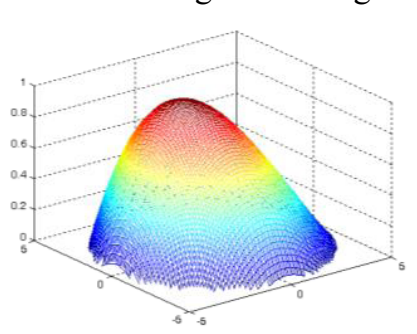

(a)

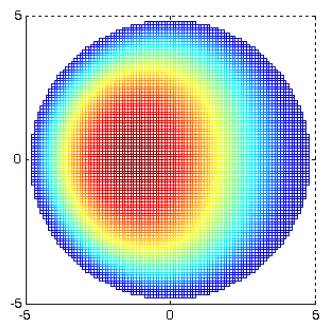

(b)
Figure 3. The normalized tool function of tilt processing mode in (a) 3D view (b) top view

\section{(2) Precession processing mode}

The precession processing mode is similar to tilt processing mode, where the bonnet rotates about its axis, as well as it rocks about the normal line of the polishing area. Since it is difficult for the bonnet to rock about its pole continuously, the near-Gaussian function is obtained by the four precession positions in practice, as shown in Figure 4 . The parameters applied in simulation is same to tilt processing mode.

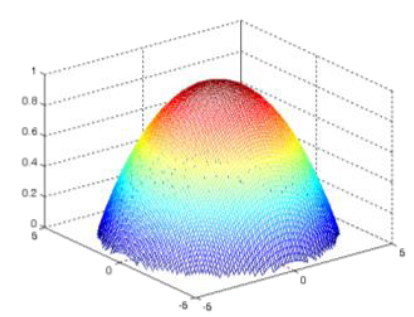

(a)



(b)
Figure 4. The normalized tool function of precession processing mode in (a) 3D view (b) top view

\section{The Dynamic FEA model for stress distribution}

In order to choose the optimal parameters during polishing, a deformation analysis over the polishing spot is calculated by means of dynamic FEA. The FEA model is shown in Figure 5. The bonnet material was ethylenepropylene-diene monomer (EPDM), and its properties are: the radius is $20 \mathrm{~mm}$, the thickness of membrane is $1 \mathrm{~mm}$, the Young's modulus is $0.1 \mathrm{GPa}$, and the Poisson's ratio is 0.49 . The default internal pressure of bonnet is $0.01 \mathrm{MPa}$. The glass is Zerodur, the Young's modulus and Poisson's ratio of which is $9.30 \times 10^{10} \mathrm{~Pa}$ and 0.30 , respectively. 


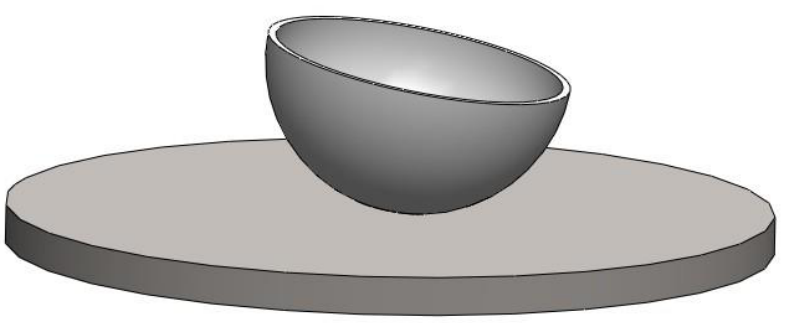

Figure 5. The FEA modal for contact deformation

\section{(1) Analysis of compression value}

The larger is the compression value, the bigger is the contact area. The compression values of $0.1 \mathrm{~mm}$ 、 $0.2 \mathrm{~mm} 、 0.3 \mathrm{~mm}, 0.4 \mathrm{~mm}$ is taken as examples, and the total stress is obtained shown in Figure 6. If the compression value is larger than $0.4 \mathrm{~mm}$, the stress distribution becomes deformed significantly. $0.3 \mathrm{~mm}$ is the best compression value.

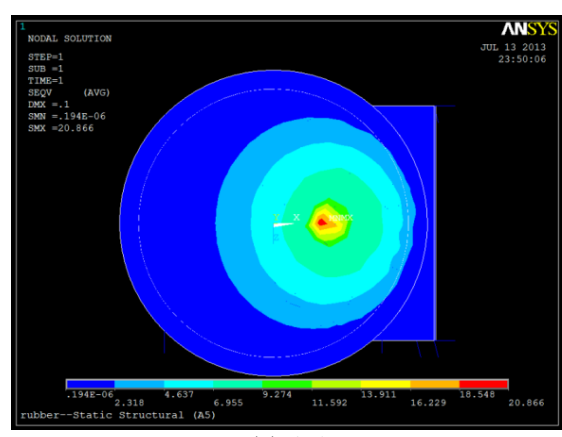

(a) $0.1 \mathrm{~mm}$

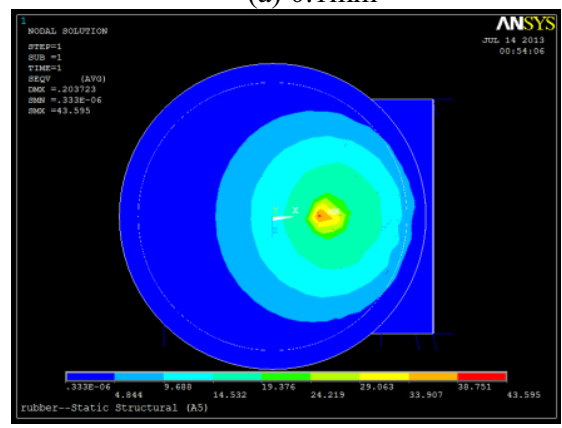

(b) $0.2 \mathrm{~mm}$



(c) $0.3 \mathrm{~mm}$

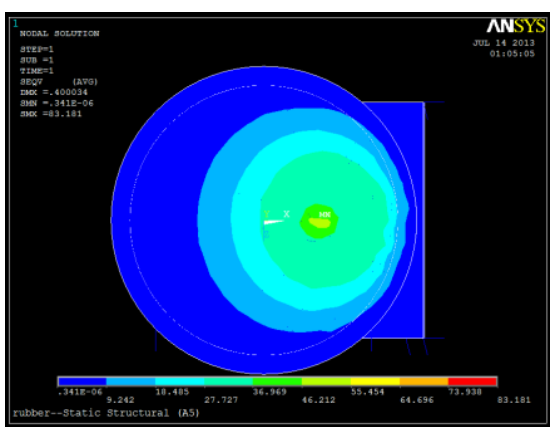

(d) $0.4 \mathrm{~mm}$

Figure 6. The total stress with different compresses

\section{(2) Analysis of rotation speed}

The total stress distributions with different rotation speeds are shown in Figure 7, which depicts that the effect of rotation speed on the tool function is not obvious. Actually, the rotation speed depends mainly on the mechanical character of CNC machine.

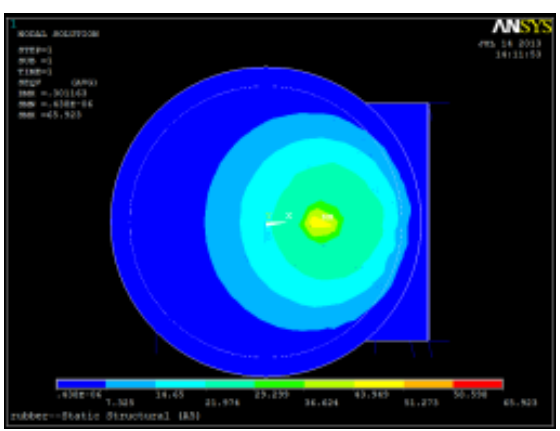

(a) $300 \mathrm{rpm}$

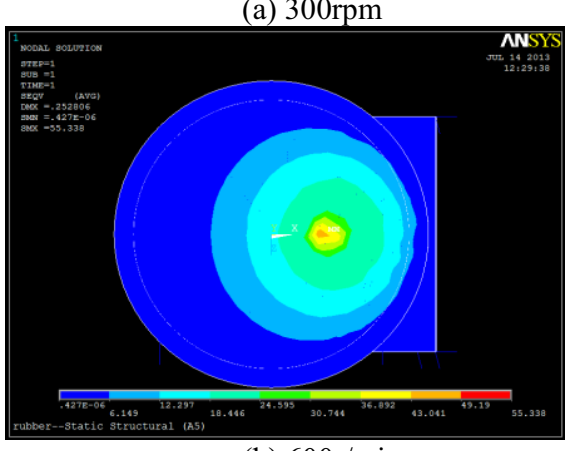

(b) $600 \mathrm{r} / \mathrm{min}$

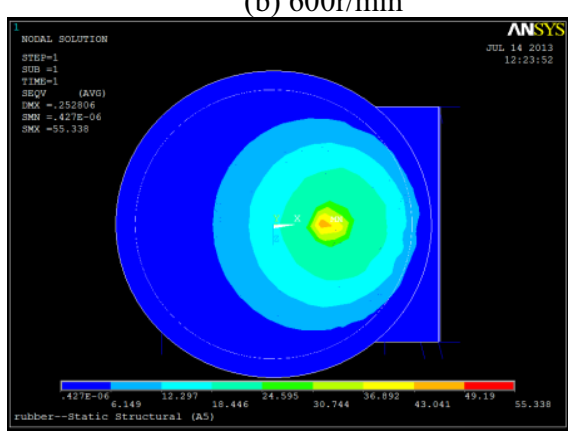

(b) $900 \mathrm{r} / \mathrm{min}$

Figure 7. The total stress with different rotation speed

(3) Analysis of internal pressure 
The internal pressure may change the elasticity of bonnet. Figure 8 depicts the total stress with different internal pressure. When the pressure is bigger than $0.05 \mathrm{Mpa}$, the outline becomes little deformed. So $0.03 \mathrm{MPa}$ was selected normally.

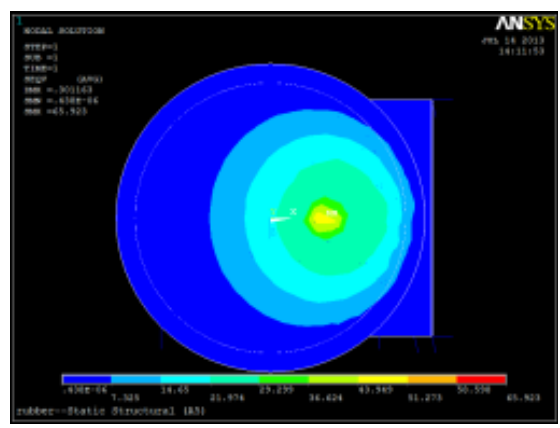

(a) $0.01 \mathrm{MPa}$

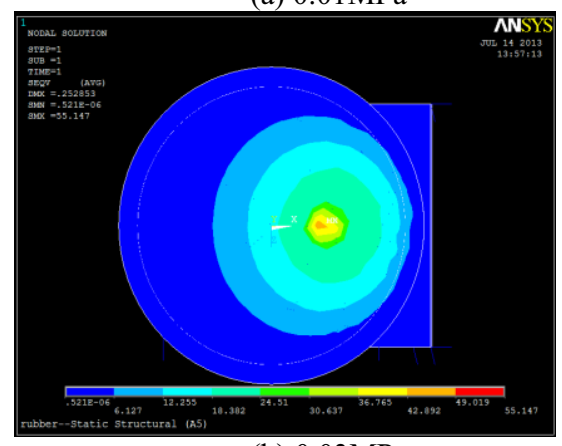

(b) $0.03 \mathrm{MPa}$

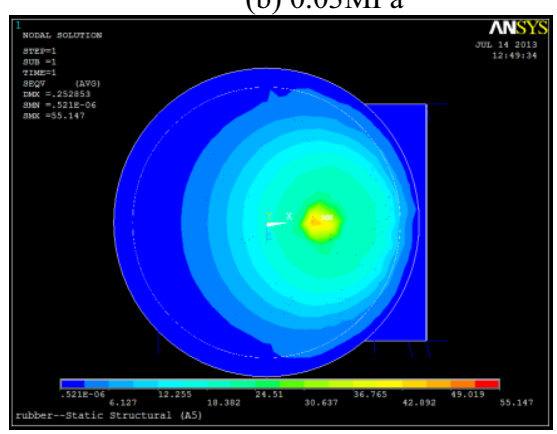

(c) $0.05 \mathrm{MPa}$

Figure 8. The total stress with different internal pressure

\section{Polishing Experimental study}

\subsection{Experimental Details}

During polishing process, some factors can affect the polishing result, such as processing time, concentrations slurry, and abrasive. A series of experiments are carried out to verify the optimal parameters.

Experimental conditions: the tilt angle is 15 degree, the abrasive is $\mathrm{CeO}_{2}$, the slurry consistency is $10 \%$, the compress value is $0.3 \mathrm{~mm}$, the rotation speed is $300 \mathrm{rpm}$, and the internal pressure is $30 \mathrm{KPa}$. The glass used is $\mathrm{K} 9$ glass. Figure 9 shows the removal depth along with the polishing time.

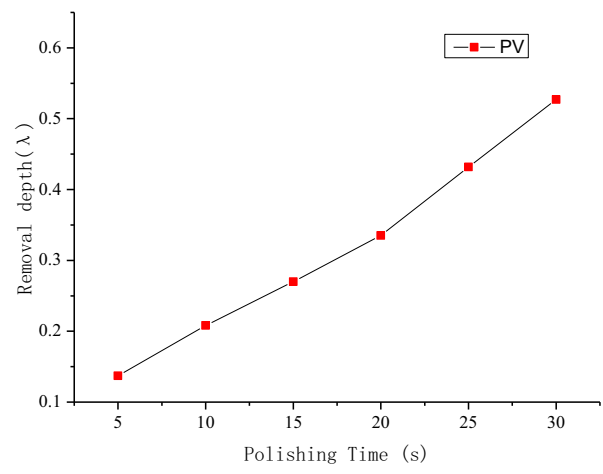

Figure 9. The removal depth along with polishing time

Next step, the removal depth with the varied concentrations of $\mathrm{CeO}_{2}$ slurry is shown in Figure 10.The result indicates that the best concentrations for $\mathrm{K} 9$ glass is about $15 \%$.

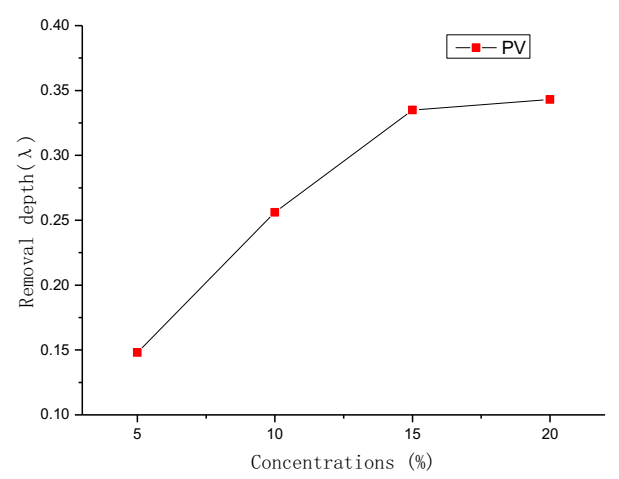

Figure 10. The removal depth with the varied concentrations of $\mathrm{CeO}_{2}$ slurry

The polishing tool with different materials have the different producing efficiency. The elasticity and hole distribution of cotton fiber, polyurethane and chammy are different. Table 1 tabulates their characters, and the Polyurethane has the best efficiency.

Table 1 The characters of different polishing clothes

\begin{tabular}{|c|c|c|c|c|}
\hline Materials & Thickness & Elasticity & $\begin{array}{c}\text { Hole } \\
\text { distribution }\end{array}$ & $\begin{array}{c}\text { Removal } \\
\text { depth }\end{array}$ \\
\hline $\begin{array}{c}\text { Cotton } \\
\text { fiber }\end{array}$ & $0.2 \mathrm{~mm}$ & Less & $\begin{array}{c}\text { about } \\
360 \times 360 \mu \mathrm{m}\end{array}$ & $0.90 \lambda / \mathrm{min}$ \\
\hline $\begin{array}{c}\text { Polyuret } \\
\text { hane }\end{array}$ & $0.5 \mathrm{~mm}$ & Small & desultorily & $1.21 \lambda / \mathrm{min}$ \\
\hline Chammy & $0.4 \mathrm{~mm}$ & bigger & no hole & $0.35 \lambda / \mathrm{min}$ \\
\hline
\end{tabular}

Actually, the material of bonnet wears after long time polishing. Empirically, the polyurethane should be replaced about every two hours, and the chammy should be replaced about every fourty minus. True, the working time depends on the polished material.

\subsection{Polishing experiment}

A $100 \mathrm{~mm} \times 100 \mathrm{~mm} \mathrm{~K} 9$ workpiece is chosen for validation of the aforementioned model and optimal parameters. During the polishing state, following parameters are used: 
the internal pressure is $0.04 \mathrm{Mpa}$, the rotation speed is $300 \mathrm{rpm}$, the procession angle is 15 degree, the compression value is $0.3 \mathrm{~mm}$, and the concentration of Cerium oxide slurry is $15 \%$. The grid path is adopted, and its pitch is $1.65 \mathrm{~mm}$. During polishing, the tilt process mode is adopted. After one running cycle about 10 minutes, the residual error decreases from PV $0.458 \lambda$, RMS $0.091 \lambda$ to PV $0.111 \lambda$ and RMS $0.016 \lambda$, as shown in Figure 11. The results indicate that the optimal parameters are correct and suitable for bonnet polishing.

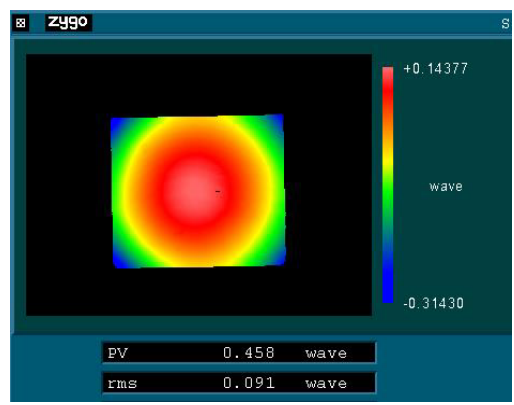

(a)

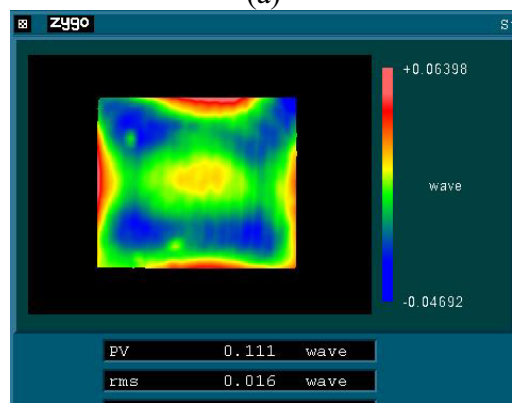

(b)

Figure 11. The residual error of polished surface (a) before and (b) after polishing

\section{Conclusions}

In this work, the removal function of bonnet polishing is modelled based on Preston hypothesis. The relative velocity distribution is obtained according to the geometry of the process tool-motion. The pressure distribution that fits parabolic distribution can be calculated through Hertzian contact theory. Then the tool function is simulated in three different modes. In this work the tilt process mode is applied. Furthermore, a dynamic FEA model is proposed to get the stress distribution between tool and optic during polishing. The optimal parameters are selected, and a series of experiments are carried on to verify the optimal parameters, for example, the internal pressure is less than $0.05 \mathrm{MPa}$, the concentrations of slurry is $15 \%$, and the working time of polyurethane should be less than 2 hours, et al. It is well known, sub-aperture technology is more prone to ripple errors, and so is the bonnet process. From the polishing experiment results, the residual surface error has some ripples, and mid-spatial frequency error is induced during polishing process. This type of surface with ripples cannot be suitable in intense laser systems and high resolution image formation systems. Further research will focus on how to diminish mid-spatial frequency errors during polishing process.

\section{Acknowledgement}

This work was supported by the National Natural Science Foundation of China (Grant Nos. 61308075, 61222506), the Specialized Research Fund for the Doctoral Program of Higher Education (Grant No. 20131101110026), Beijing Natural Science Foundation(Grant No. 4144083), and Excellent young scholars Research Fund of Beijing Institute of Technology.

\section{References}

1. R. A. Jones, Photon. Spectra, 34-39 (1963).

2. R. E. Wagner and R. R. Shannon, Appl. Opt. 13,7, (1974).

3. R. A. Jones, Opt. Eng. 22 (1983).

4. D. D. Walker, D. Brooks, A. King, R. Freeman, R. Morton, G. McCavana, and S. W. Kim, Opt. Express 11,8 (2003).

5. J. H. Burge, S. Benjamin, D. Caywood, C. Noble, M. Novak, C. Oh, R. Parks, B. Smith, P. Su, M. Valente, and C. Zhao, Proc. of SPIE 7426 (2009).

6. D. W. Kim, S. W. Kim, and J. H. Burge, Opt. Express 17,24 (2009)

7. M. Demmler, M. Zeuner, et al. Proc. of SPIE 7934, 793416 (2011)

8. R. Jourdain, M. Castelli, P. Morantz, P. Shore. Proc. of SPIE 8430843011 (2012)

9. W. Kordonski , S. Gorodkin, Appl. Opt. 50 ,14 (2011)

10. W.I. Kordonski, A.B. Shorey, M. Tricard, , J. Fluid Eng-T ASME, 128,1 (2006)

11. T. Wang, H. Cheng, H. Yang, W. Wu, H. Tam. Appl. Opt. 54, 19,(2015)

12. D. D. Walker, R. Freeman, R. Morton,G. McCavana, A. Beaucamp, Opt. Express, 14,24 (2006).

13. D. Walker, G. Yu, H.Li, W. Messelink, R. Evans, A. Beaucam. Opt. Express. 20,18 (2012)

14. H. Li,.D.Walker, G. Yu. A.Sayle, W. Messelink, R. Evans, A. Beaucamp, Opt. Express 21,1 (2013)

15. F. W. Preston, J. Soc. Glass Technol. 11 (1927) 